\title{
Research on Embedded Electrical Impedance Measurement System
}

\author{
Xiao-yan $\mathrm{CHEN}^{1, \mathrm{a}}$, Liang SUN ${ }^{2, \mathrm{~b}^{*}}$ and Meng DU $\mathrm{U}^{3, \mathrm{c}}$ \\ ${ }^{1,2,3}$ College of Electronic Information and Automation, Tianjin University of Science and \\ Technology, 300222, Tianjin,China \\ acxywxr@tust.edu.cn, ${ }^{\mathrm{b}}$ Liangsun9010@163.com, ${ }^{\mathrm{c}} \mathrm{mdu} @$ tust.edu.cn
}

Keywords: ARM processor, AD5933, QT, Impedance tomography.

\begin{abstract}
Based on S3C2440 processor and Linux3.6.6 kernel, wedeveloped an embedded impedance measurement system. This embedded system employ AD5933 as the impedance sensor, and data communication between AD5933 and S3C2440 processor is implemented with IIC communication protocol. In addition, the measurement resultsare displayed on the interface that is developed with QT.After modeling and solving the inverse problem by Newton Gauss algorithm, the distribution images of the object in the field are finally reconstructed satisfactorily. The research work offers a feasible and portable solution for clinical application of digital medical technology.
\end{abstract}

\section{Introduction}

Biomedical electrical impedance tomography (BEIT), as a newly noninvasive medical imaging technique, has been attracted much attentions in the field of biomedical engineering [1,2,3]. Compared to traditional imaging techniques, it has many advantages such as low cost, reusable, non-destructive, et al[4]. The BEIT technique which can monitoring the impedance variations between different tissues and organs, provides a new way to diagnose diseases [5,6,7,8].

Currently, the data acquisition module of most impedance measurement system are implemented with field-programmable gate array(FPGA)or digital signal processor(DSP)or PCI extensions for Instrumentation(PXI) and the collected experimental data is send to the computer with USB or serial port to reconstruct the images of the diseased tissue[9,10,11]. However, it quite inconvenient for moving these equipment when displaying the reconstructed images on computer monitors. It doesn't come near to the standard of current intellectualized medical instrument. In this regard, we in this paper develop aportable system that used for bioelectrical impedance measurement. Arm processor and Linux system are integrated into this embedded system. Impedance data are collected at the measuring end of this system, and the collected data are displayed with QT platform. This portable embedded based impedance measurement system integrate are more convenient and efficient when used for diagnosing diseases.

\section{Hardware Design}

\section{System Structure}

The system structure of this portable system is shown in Fig.1. As we can see, the communication between the S3C2240 and AD5933 is implemented with the $\mathrm{I}^{2}$ Cprotocol. The impedance data are measured and displayed with manipulating the 
buttons on the touch screen. Also, the data curves can be analyzed and displayed with QT platform.

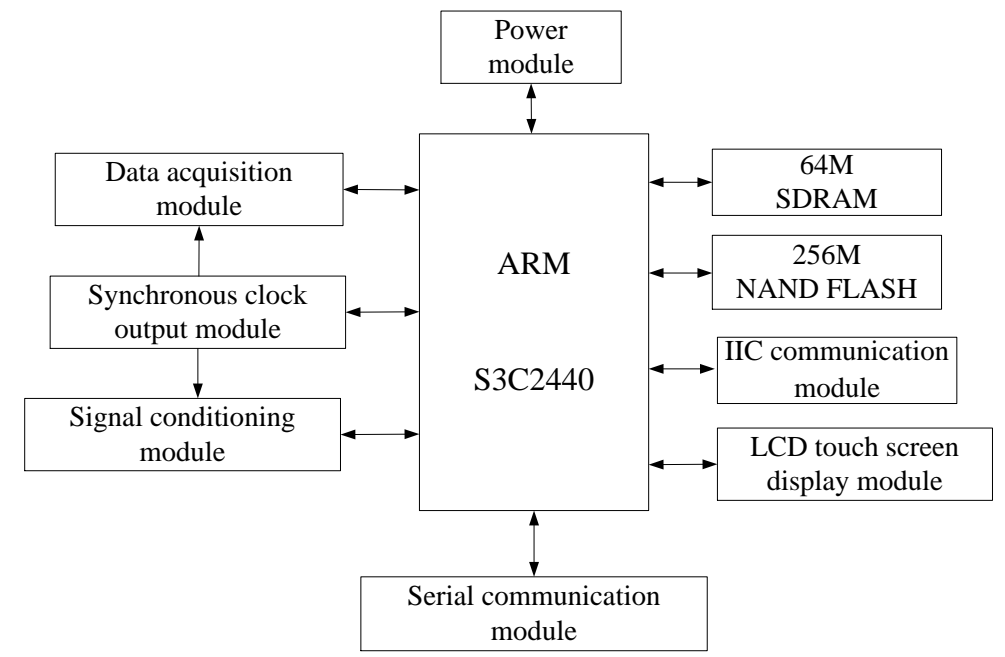

Fig. 1 System structure of the impedance measuring system.

With this framework, we construct an eight electrode embedded EIT system. the system main controller controls each electrode with IIC bus, while each electrode employa single AD5933 to achieve the excitation and measurement. This embedded system include a power module, a signal processing module, a IIC communication module, a data acquisition module, a synchronous clock module and a serial communication module.

\section{Signal Conditioning Module}

The sinusoidal exciting signal is generated with the phase accumulator (DDS) in AD5933 and excited into the measurement field. Then the measured voltage are collected by using the on-board analog-to-digital converter (ADC) and processed with discrete Fourier transform (DFT)algorithm to obtain the real part (R) and imaginary part $(\mathrm{R})$ of the collected signals. According to the manual, when choose the $1.98 \mathrm{Vp}-\mathrm{p}$ the output DC bias is $1.48 \mathrm{~V}$, while, the current voltage (I-V) amplifier bias voltage of $\mathrm{AD} 5933$ is $\mathrm{VDD} / 2=1.65 \mathrm{~V}$. In this regard, the existed phase difference would lead to inaccuracy of the measurement. To solve this problem, we use the following strategy to eliminate this affection. First, we pre-process the output voltage by using a high-pass filter to eliminate the affection of DC component which would keep the same DC bias between measurement and excitation. Also, we design the voltage follower by using a low output resistance COMS amplifier AD8606 to eliminate the affection of output impedance. Furthermore, we improve the accuracy of I-V conversion by using a external I-V converter. This design not only eliminates the error involved by polarization, but also enlarges dynamic range of the internal ADC which would greatly improve the performance of the circuit.

\section{Data Acquisition Module}

We in this paper employ an eight electrode impedance measurement system.During a sampling period, one of the eight electrodes is excited and the experimental data are collected from the other 7 electrodes. Take electrode 1 as exciting electrode for example, the other 7 electrodes are assigned measurement electrode. During the nest sampling period, electrode 2 is assigned as exciting electrode and the other as measurement electrode, and so on. After all the 8 electrodes are excited, a frame of data collection is finished which include 56 data section. So, one frame data collected 
by this impedance measurement system include $\mathrm{N}^{*}(\mathrm{~N}-1)$ date sections, where $\mathrm{N}$ denotes the number of electrodes in the impedance measurement system. These collected frames can be used for image reconstruction. Fig. 2 shows the schematic of the 8 electrode impedance measurement system.

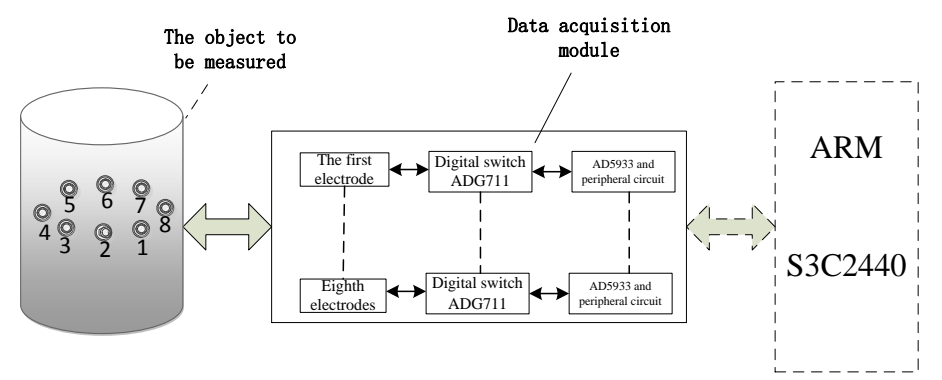

Fig. 2 Schematic of the 8 electrode impedance measurement system.

\section{Synchronous Clock Module}

In order to ensure the synchronization during the data collection, we use AD9520-5 chip to generate the synchronous clock. There are two working modes for AD9520-5, i.e. low frequency mode and high frequency mode [12]. We choose the low frequency modein the senses that the typical frequencyof AD5933 internal clock is 16.776 MHz.There is no internal Vector-Controlled Oscillator (VCO) in AD9520-5, so we use external crystals and set the frequency coefficients to obtain the oscillation which can ensure the synchronization.

\section{Software Design}

The system operation process is as follows: first,the system is initialized andlogged into the operation interface of QT platform. Then system parameters such as scanning parameters, clock frequency are set on the operation interface.After that electrode working mode is also chosen.Execute the command to control AD5933 for collecting the experimental data.The measured data are saved in the system flash.Fig.3 shows the flow chart of the main program of this system.

Slot is a function that can be called to deal with a particular signal. Also, QT can be added to a custom slot. As shown in Fig.4, based on the added custom slot, we develop a system user interface to deal with the signals more efficiently. As shown in Fig.5, we can click the 'AD933set' button to complete the initialization process.It is worth nothing that due to the different settings and amount of collected data, the system initialization time will be different.

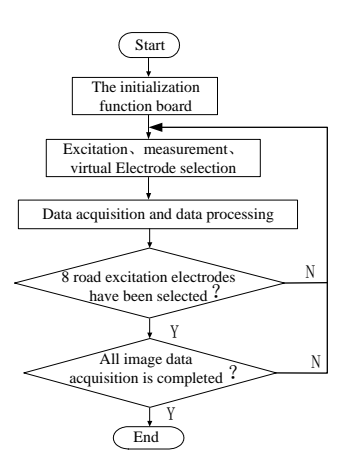

Fig. 3Main program flow

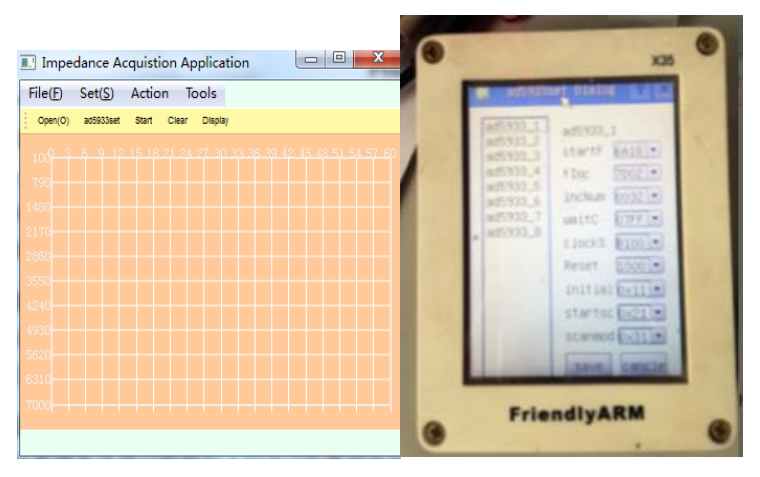

Fig.4 QT interface
Fig.5 AD5933 initialization 


\section{Experime ntal Verification}

As shown in Fig.6, the object to be measured is a $15 \mathrm{~cm}$-diameter $25 \mathrm{~cm}$-high plexiglasscylindrical tank which filled with saline. The conductivity of the filled saline is $0.36 \mathrm{~S} / \mathrm{m}$, and the ambient temperature is $15^{\circ} \mathrm{C}$. The imaging target is a $4 \mathrm{~cm}$ diameter cylindrical plexiglass rod. Eight $4 \mathrm{~mm}$ titanium metal electrode are uniformly distributed around the inner side of the tank.

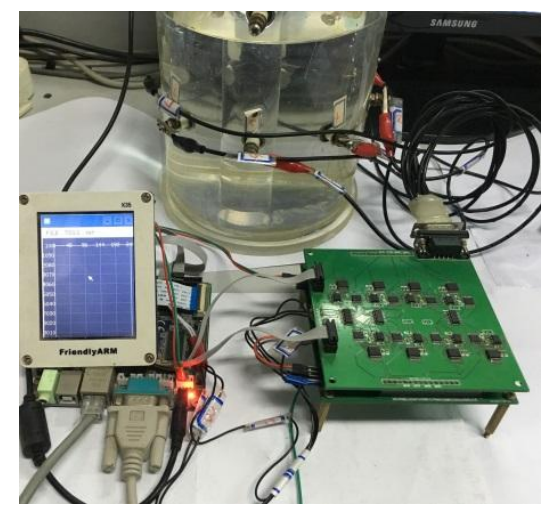

Fig. 6 Measurement system

Under the COMSOL environment, a simulation model is established to calculate amplitude and sensitivity matrix. Newton Gauss algorithm is adopted for the image reconstruction. We use four images in different locations to demonstrate the imaging quality.

Table1 Experimental reconstruction results

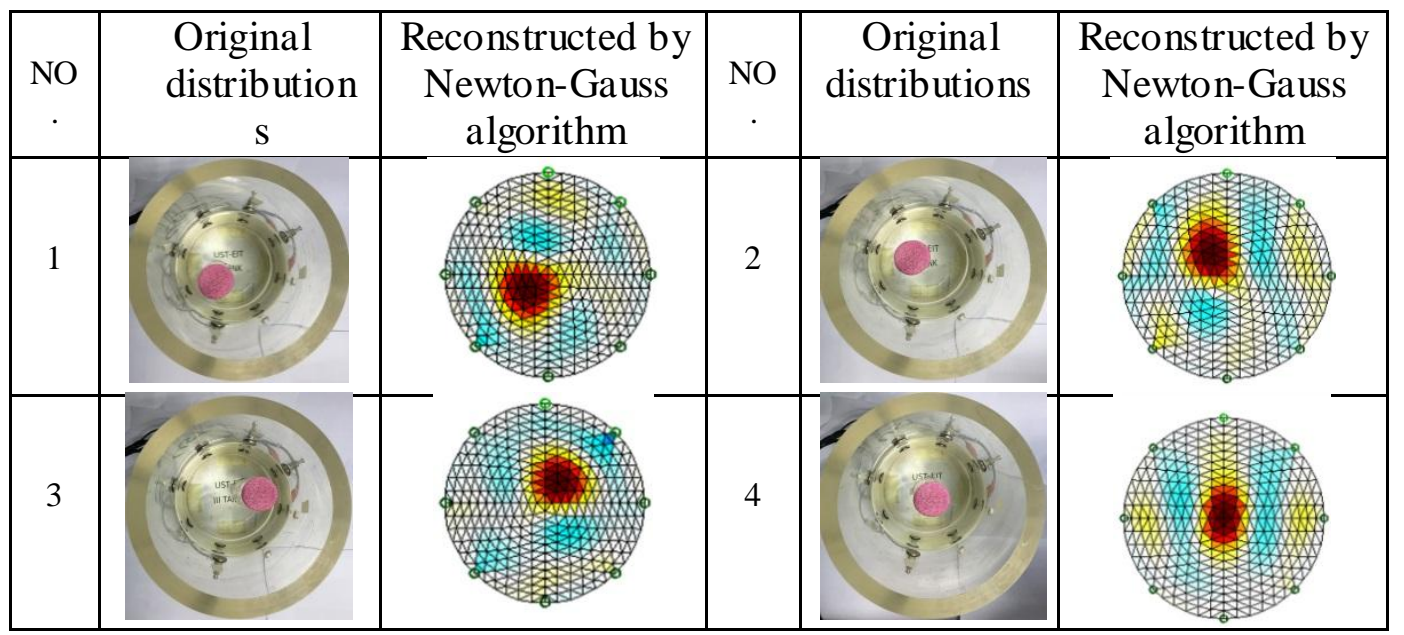

As can be seen from the table 1, the embedded impedance measurement system can accurately demonstrate the shape, size and position of the organic glass rod.

\section{Conclusions}

In this paper, the measurement and displaying is realized with the embedded impedance measurement system of eight electrodes. After modeling, solving and reconstructing the image algorithm, the distribution of the object in the field is finally obtained. In addition, the system can also use the AD5933 to sweep frequency. Select two electrodes to work independently and set $100 \mathrm{KHz}$ for two port impedance data measurement. Due to the constraint of memory and computing resources of S3C2440 processor, it is difficult to implement complex reconstruction algorithm on this 
embedded system. With the development of embedded technology, it is expected that the function of measurement, display and imaging can be integrated into one.

\section{Ackno wledge ments}

This work was financially supported by the Natural Science Foundation of China (61301246, 61302122) and the Natural Science Foundation of Tianjin Municipal Science and Technology Commission (12JCYBJC19300).

\section{References}

[1]. Barber D C; Brown B H.Applied Potential Tomography[J]. Journal of Physics E: Scientific Instruments, 1984, 17(9):723-733.

[2]. HalterRJ;HartovA; PaulsenKD. Transactions on biomedical engineering ,IEEE 2008, 55(2): 650-659

[3]. Chen X Y;Wang H X; Zhao B; Shi X L. Lung Ventilation Functional Monitoring Based on Electrical Impedance Tomography. Trans. Tianjin Univ, 2009, 15: 007-012.

[4]. Zarafshani A, Huber N; BeqoN;Tunstall B ;Sze G ;Chatwin C ; Wang Wei. A flexible low-cost, high-precision, single interface electrical impedance tomography system for breast cancer detection using FPGA[J]. ICEBI and EIT 2010, (1)

[5]. Zhang Jie;Patterson, Robert. Variability in EIT images of lung ventilation as a function of electrode planes and body positions[J]. Open Biomedical Engineering Journal, 2014, (1): 35-41

[6]. Leonhardt Steffen; Pikkemaat Robert; Stenqvist Ola; Lundin Stefan. Electrical Impedance Tomography for hemodynamic monitoring[J]. Annual International Conference of the IEEE Engineering in Medicine and Biology Society, 2012, 122-125

[7]. TrokhanovaOV;Chijova, YA;Okhapkin, M B; Korjenevsky AV ;Tuykin T S. Using of electrical impedance tomography for diagnostics of the cervix uteri diseases [J]. International Conference on Electrical Bioimpedance, 2010, 224(1)

[8].Leonhardt Steffen; Pikkemaat Robert; Stenqvist Ola; Lundin Stefan. Electrical Impedance Tomography for hemodynamic monitoring[J]. Annual International Conference of the IEEE Engineering in Medicine and Biology Society, 2012, 122-125

[9]. Khan, S.; Borsic, A. ; Manwaring, Preston. FPGA based high speed data acquisition system for electrical impedance tomography[C].Journal of Physics: Conference Series:Institute of Physics Publishing, 2013

[10].GoharianMSoleimaniM,Jegatheesan A.A DSP Based Multi-Frequency 3D Electrical Impedance Tomography System[J].Annals of Biomedical Engineering, 2008, 36(9):1594-1603.

[11].Chen X Y,YangT,Yang Y Z.A PXI-Based Biomedical Electrical Impedance Tomography System[C].Applied Mechanics and Materials, 2014, 670-671: 1205-1209.

[12] Information on http://www.analog.com/ 